\title{
A High-Efficiency and High- Resolution Straw Tube Tracker for the LHCb Experiment
}

Niels Tuning

NIKHEF Amsterdam, The Netherlands

On behalf of the LHCb Outer Tracker Collaboration

1) Detector Construction

2) Beam Test Results
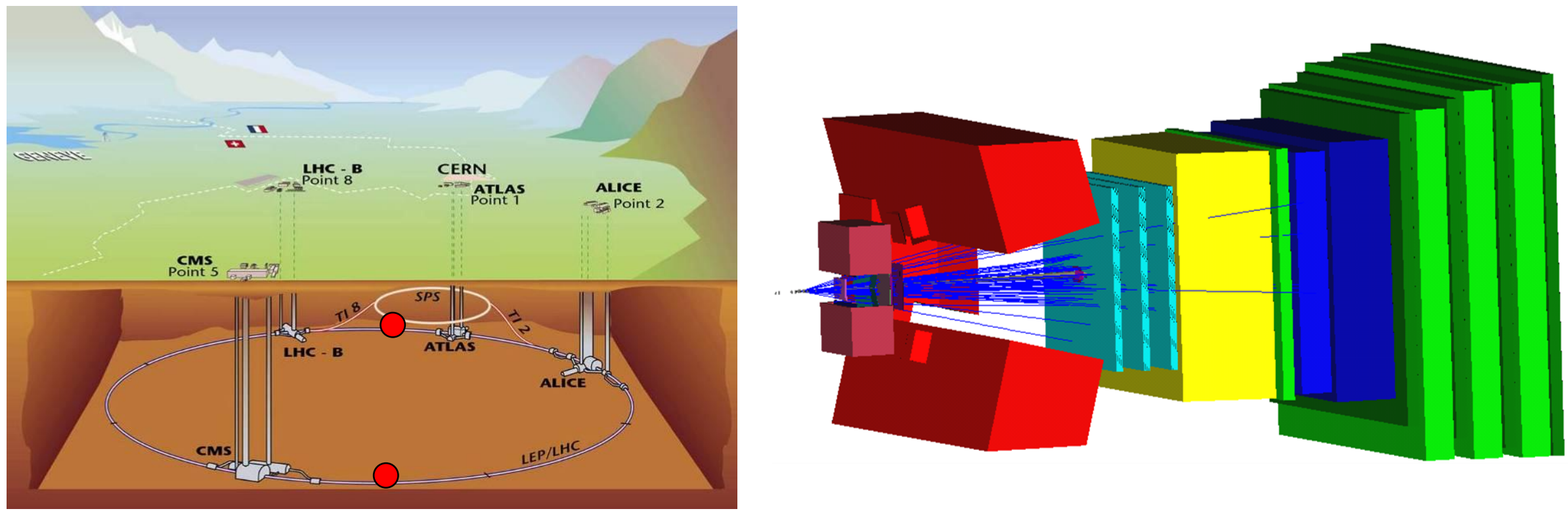


\section{LHCb in a nutshell}

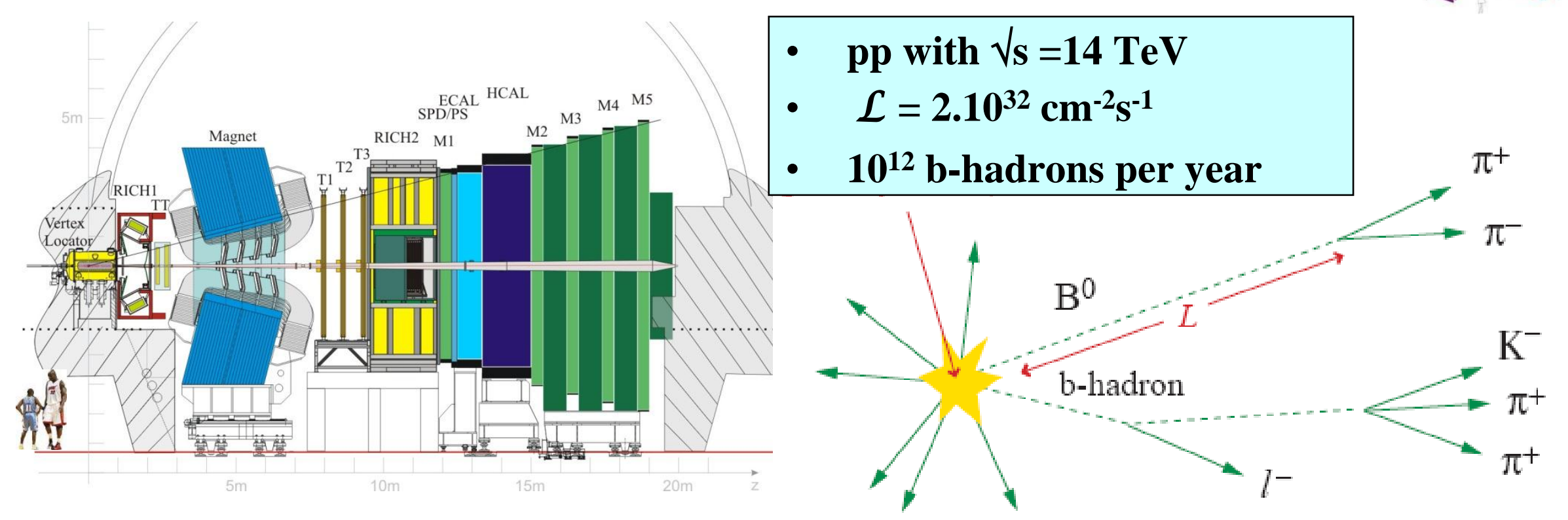

Both b's are produced forward:

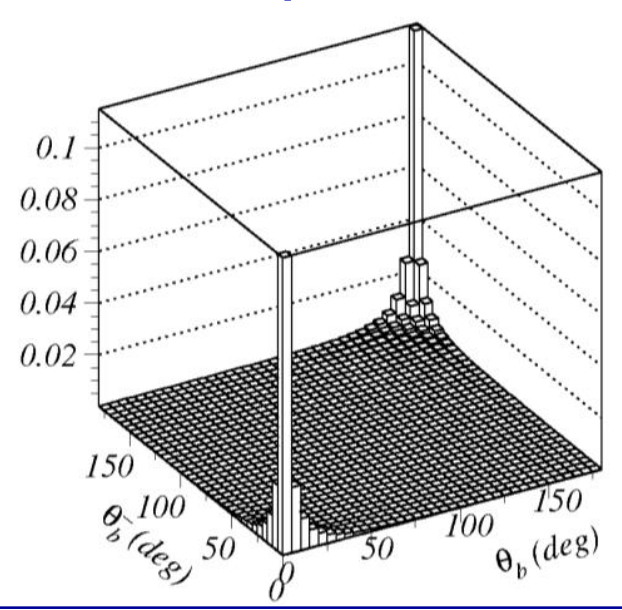

$\left.\begin{array}{l}\text { Aim: measure CP violation and rare } \\ \underline{\text { decays }} \\ \text { - } \mathrm{B}_{\mathrm{s}} \text { mixing } \\ \text { - CKM angle } \mathrm{Y} \\ \text { - Small branching fractions } \\ \text {-... }\end{array}\right\} \begin{aligned} & \text { All affected by } \\ & \text { new particles } \\ & \text { inside loops! }\end{aligned}$




\section{The LHCb experiment}
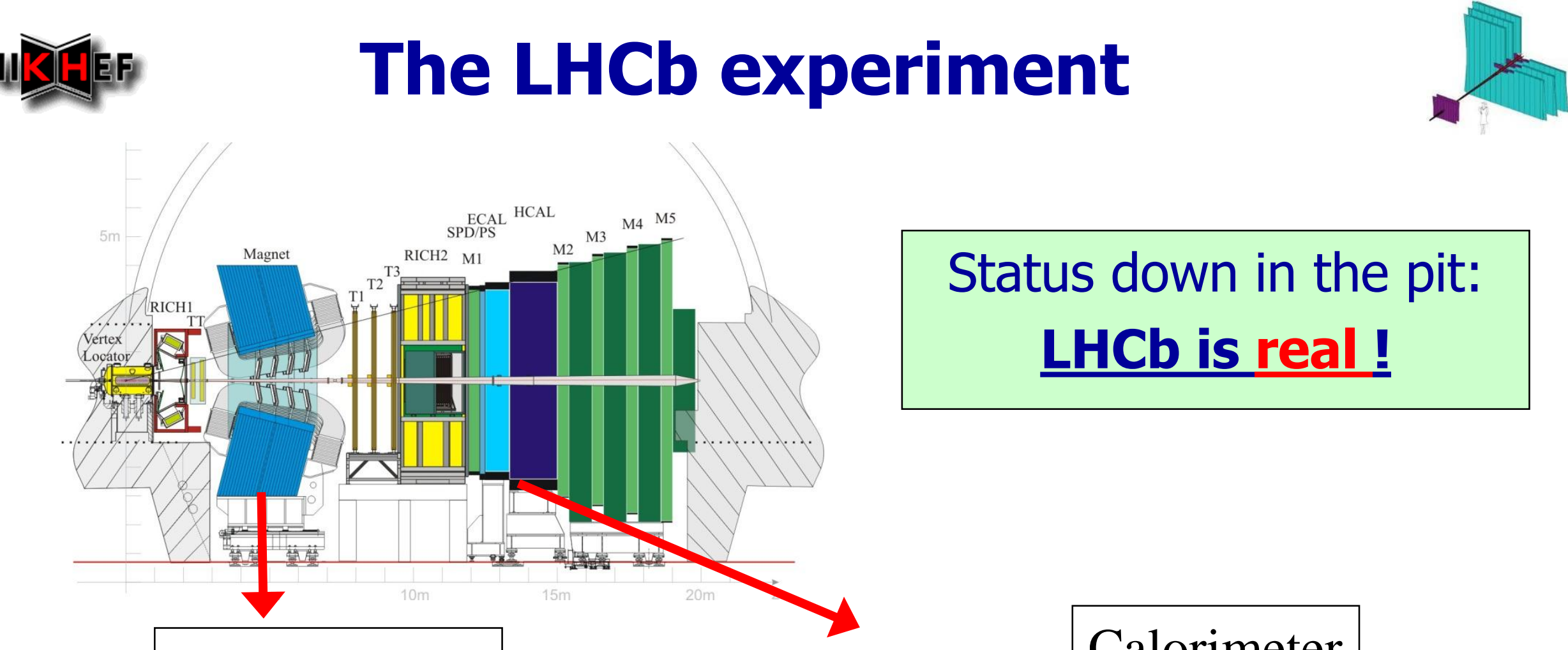

Status down in the pit: LHCb is real!

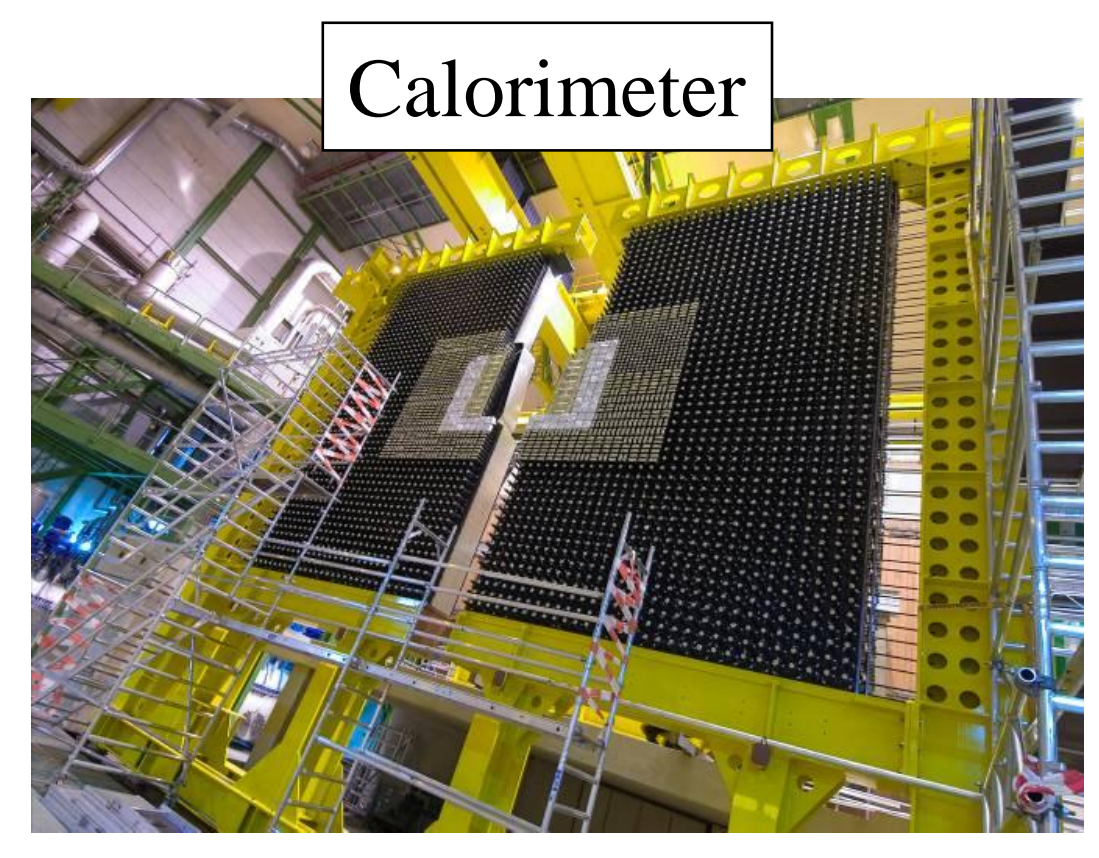




\section{LHCb experiment: The Outer Tracker}

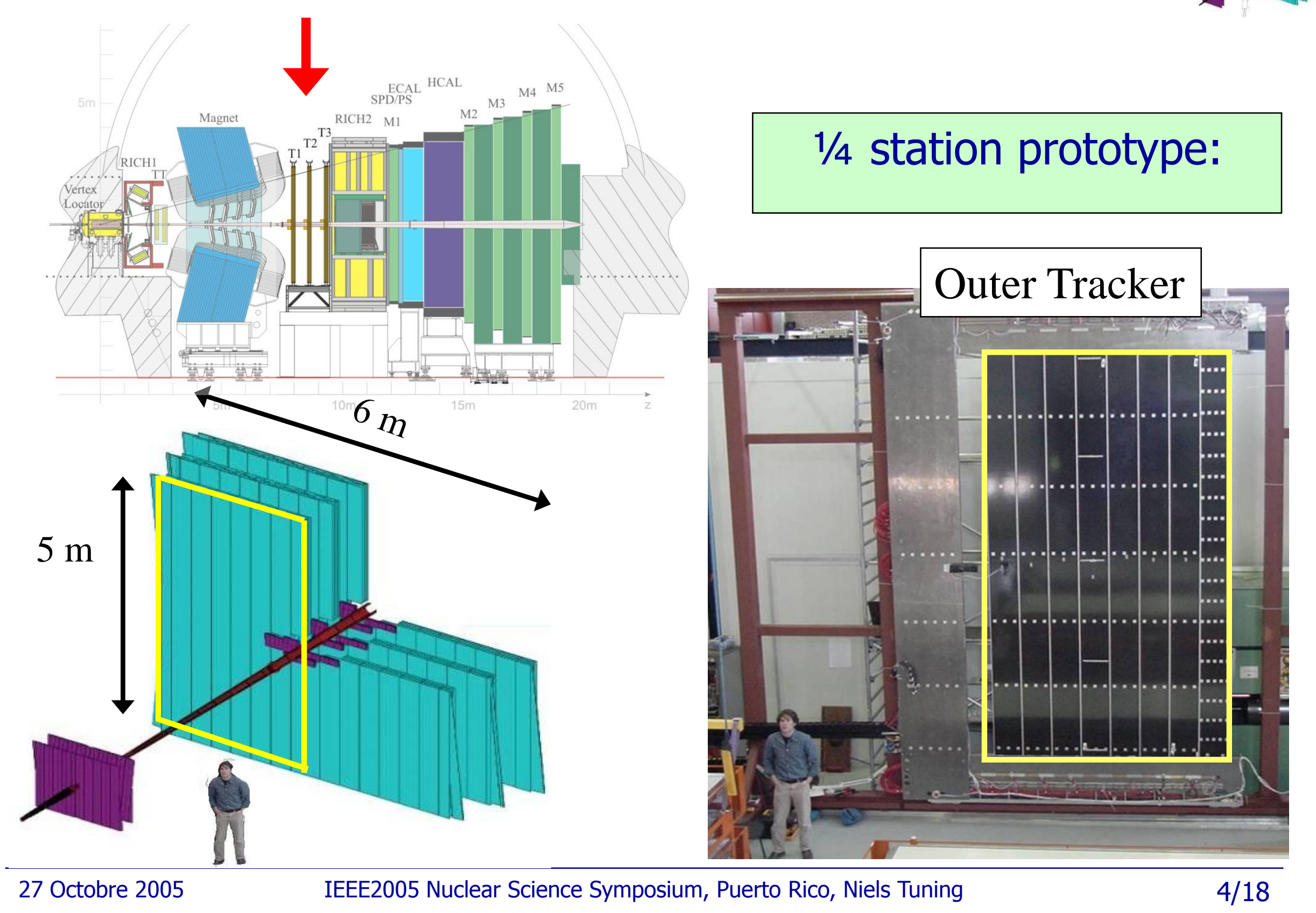




\section{The Outer Tracker}

- Cracow: all straw-support panels

- Warsaw: 124 short modules (6 types)

- Heidelberg: 62 long modules

- NIKHEF: 130 long modules

\section{Two Halves:}

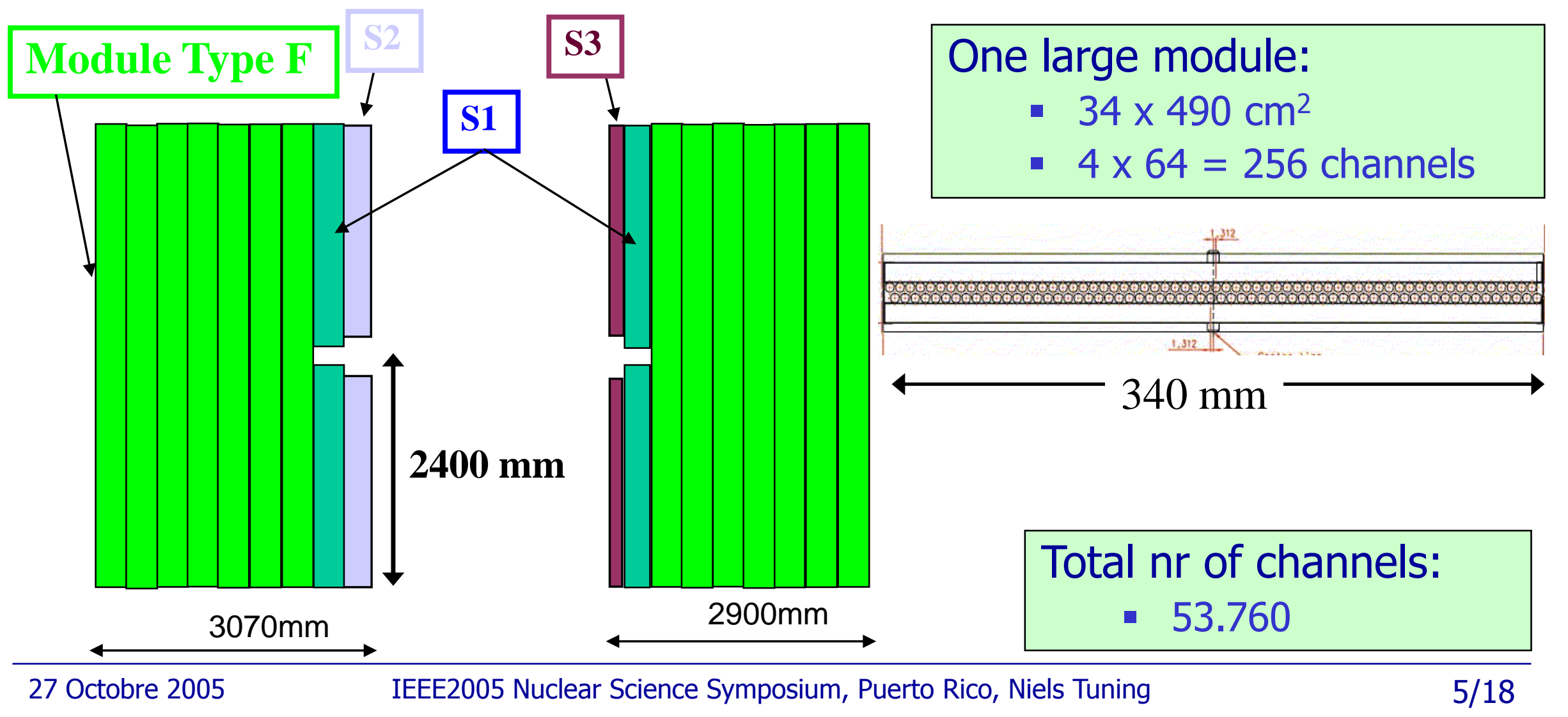




\section{Outer Tracker: Detector and Electronics}

- Straw:

- Radius: $2.5 \mathrm{~mm}$

- Length: $2.5 \mathrm{~m}$

- Gas:

- $\mathrm{Ar} / \mathrm{CO}_{2} 70 / 30$, flush at 1 volume/2 hr

- Drift time: 45 ns for $2.5 \mathrm{~mm}$

- Electronics:

- ASDBLR chip:

- Amplifies, shapes and discriminates

- Profit from ATLAS TRT

- OTIS chip:

- Measure time with accuracy < 1ns

- Gigabit Optical Link (GOL)

- Serializes and ships data at $1 \mathrm{MHz}$
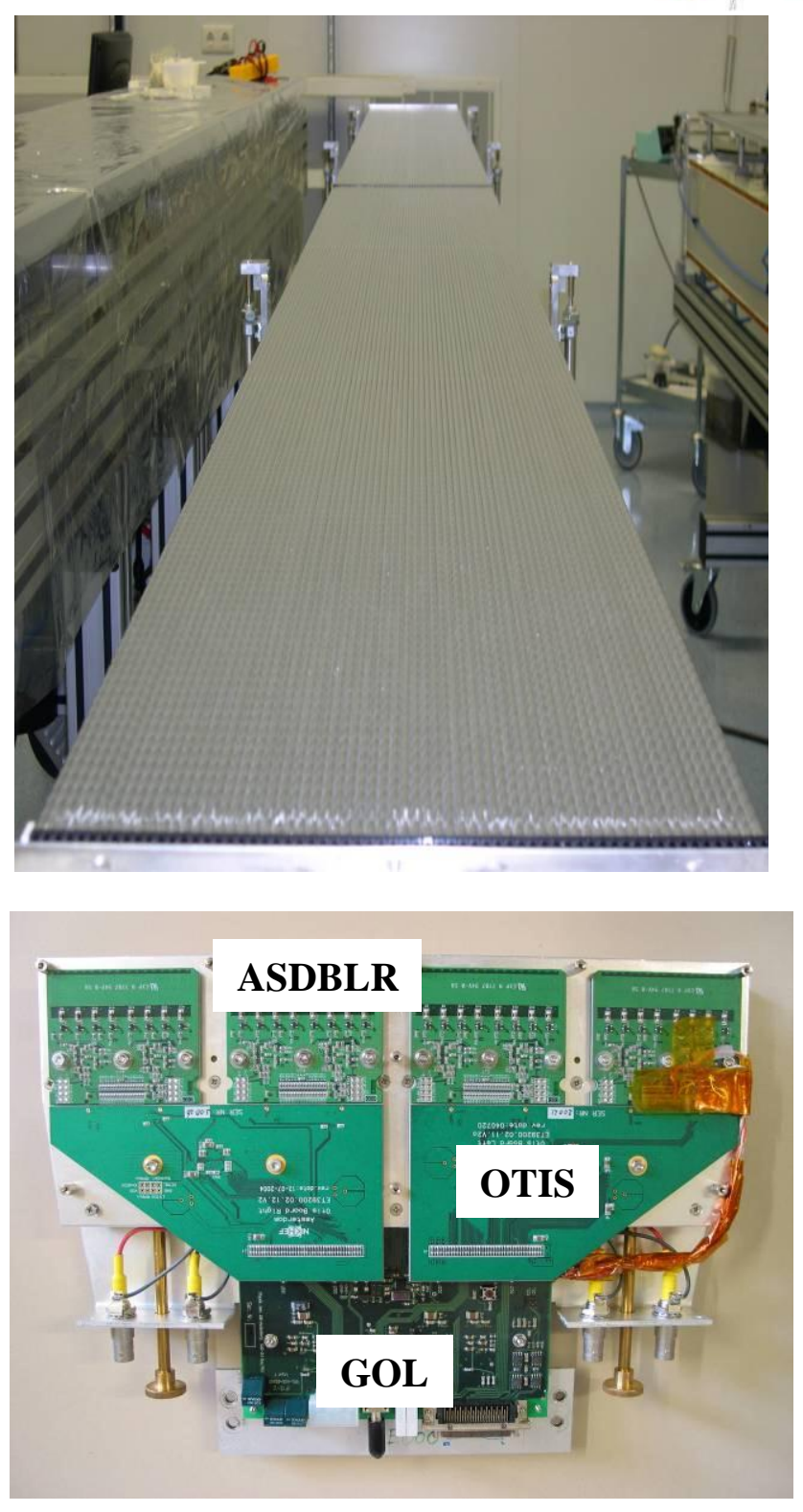


\section{OT Module Production}

\section{1) Cutting Straws}
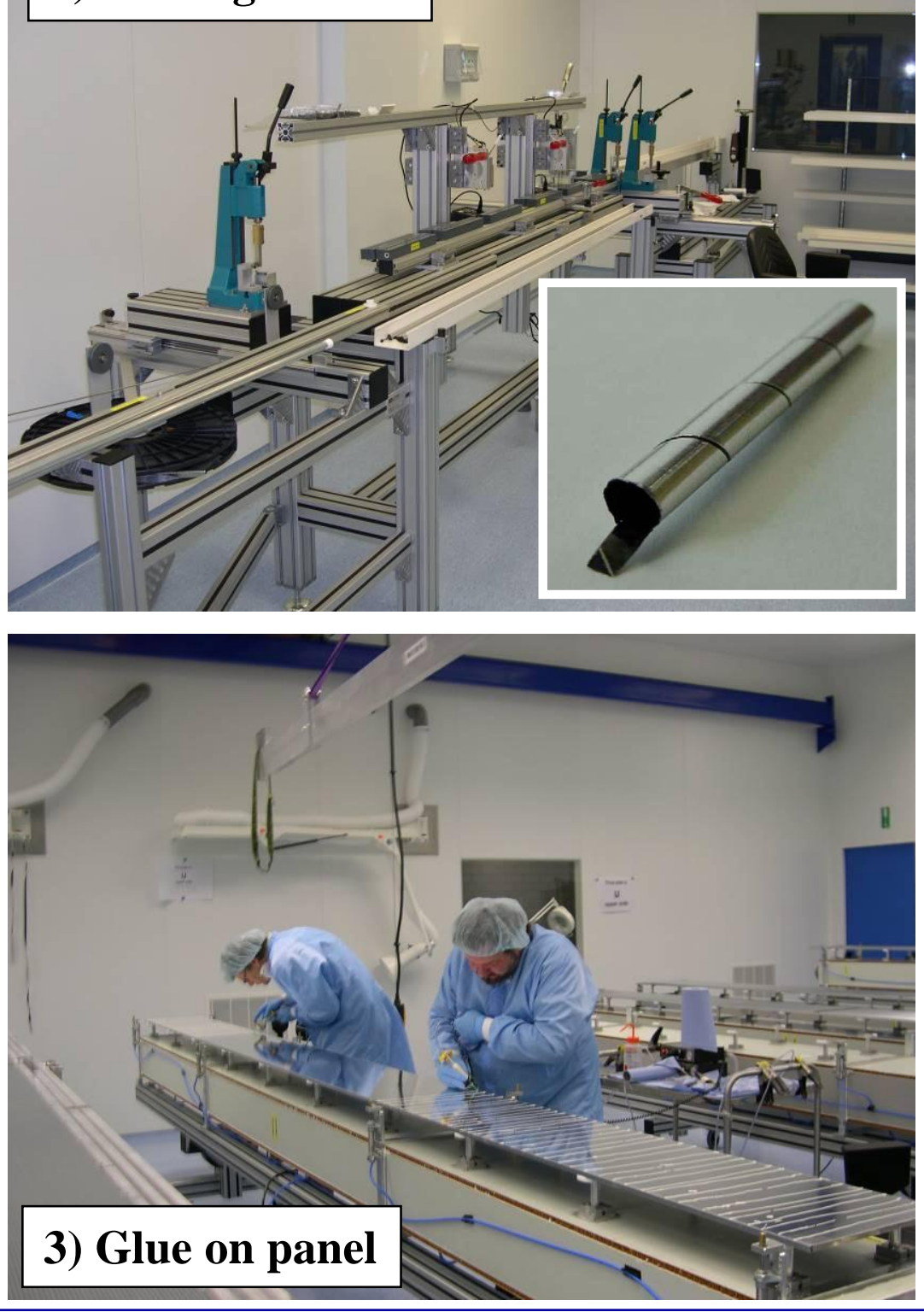
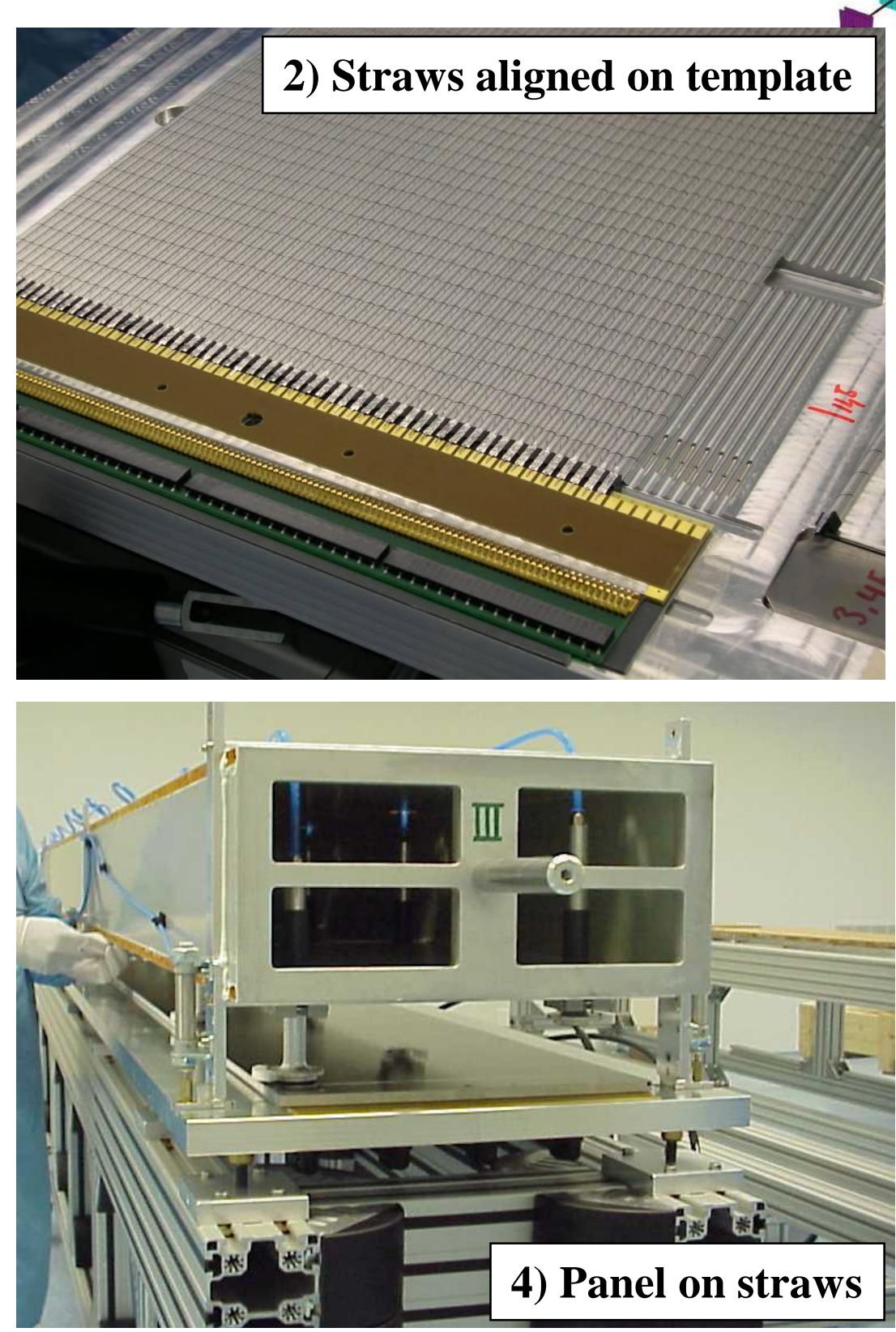


\section{OT Module Production: wiring}

- Wire quality was problem

- Wire: W/Au

- Curliness: difficult for the wiring

- Gold layer: high currents

- Rejection caused tight delivery schedule, but good wire quality eventually for all modules
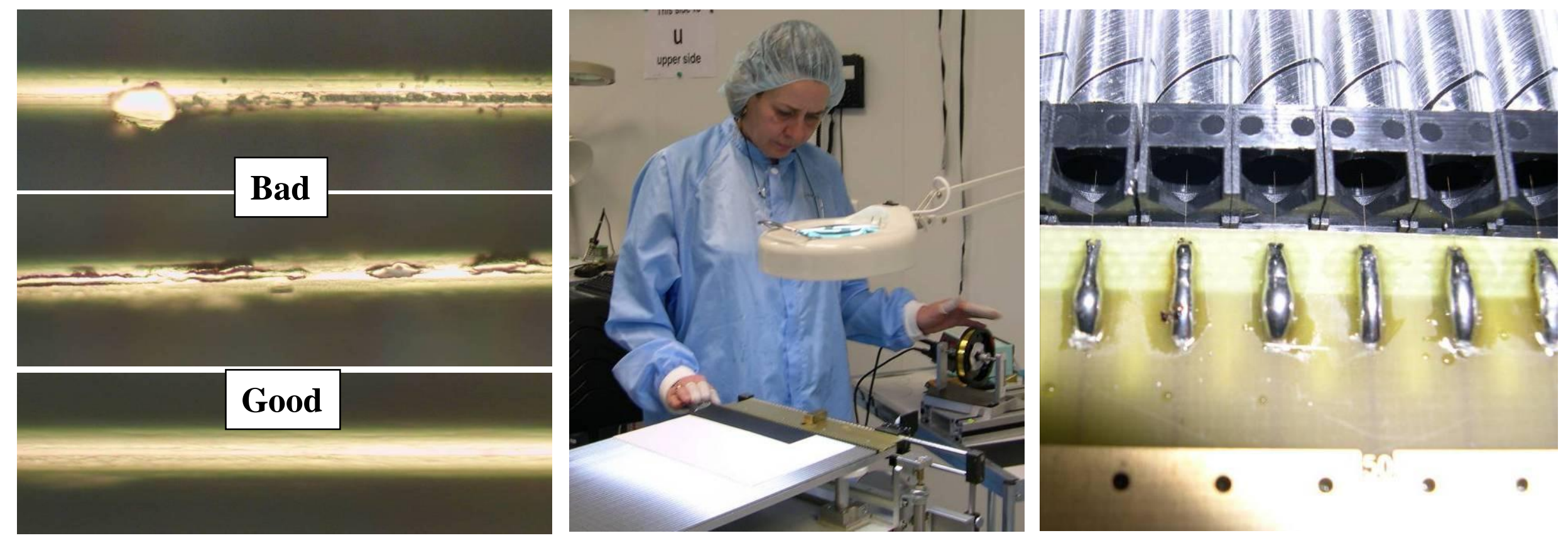


\section{OT - Quality Assurance}

\begin{tabular}{|c|c|}
\hline \multirow{2}{*}{\multicolumn{2}{|c|}{ Per Wire: }} \\
\hline & \\
\hline - Wire Tension: & $70 \mathrm{~g}$ \\
\hline - Dark Current & $<2 \mathrm{nA}$ \\
\hline Per Module: & \\
\hline $\begin{array}{l}\text { - Gas Tightness: } \\
\text { - Response to sc }\end{array}$ & $\begin{array}{l}<10^{-4} \text { I/s } \\
\text { e: } \pm 10 \%\end{array}$ \\
\hline
\end{tabular}

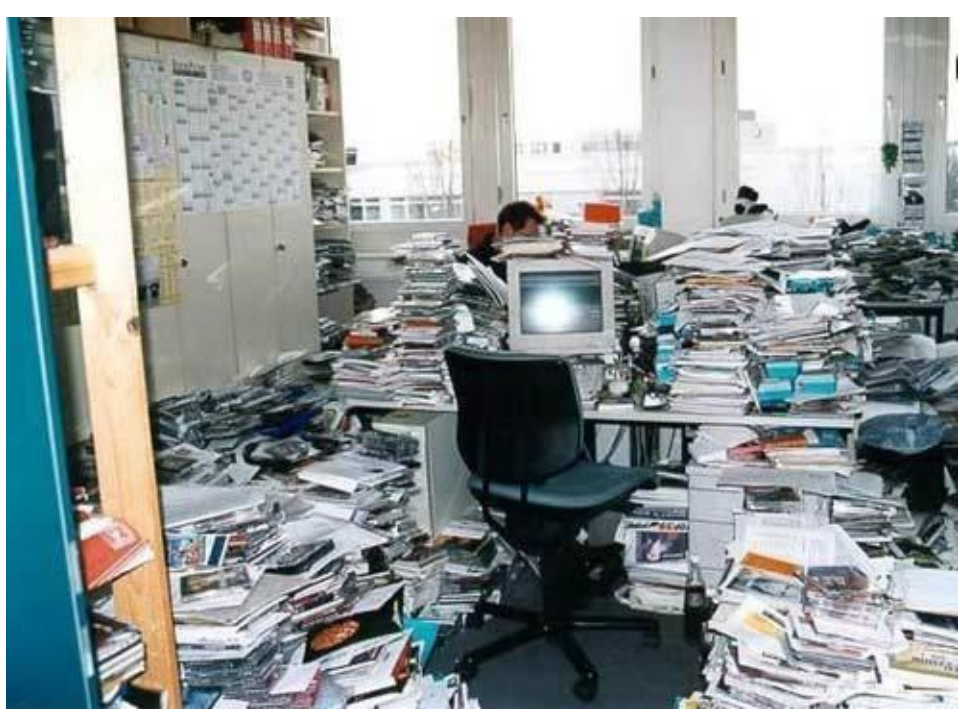

Findings:

- Bad wire quality

- Leaky gluing procedure

- Bad gas flow in outer straws

- Bad soldering of connectors

- Missing wire locators

- ...
Spotted timely

$\rightarrow$ Adapted

Procedure

$\rightarrow$ All solved!
Total number of bad channels:

- Dead, tripping or noisy

- $<1 \%$ o 


\section{Final module check: radio-active sources}

Check detector response:

- Current from ${ }^{90} \mathrm{Sr} \beta$-source

- Pulse height from monochromatic ${ }^{55} \mathrm{Fe} \gamma$-source

- Full scan of the entire module

- Every $\mathrm{cm}^{2}$ of the OT is checked

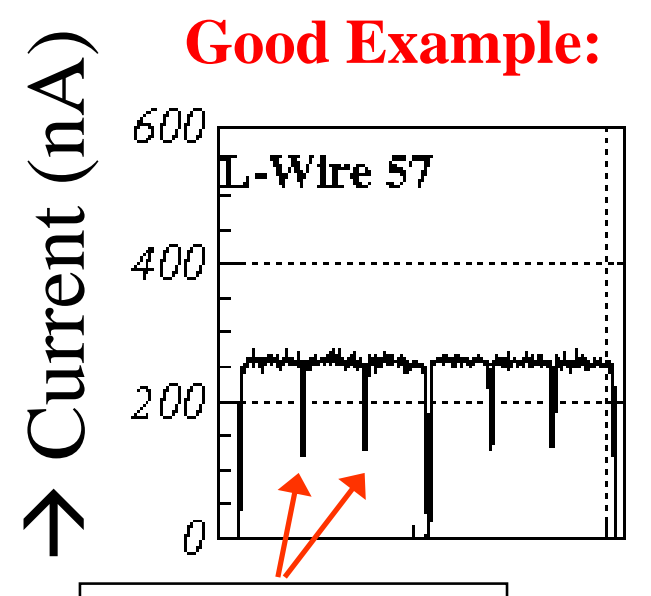

Wire locators
Bad Example:

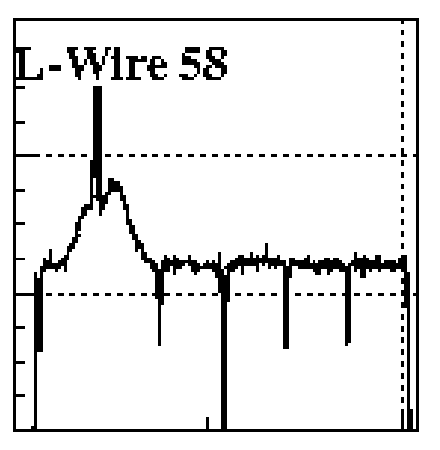

$\rightarrow$ Position

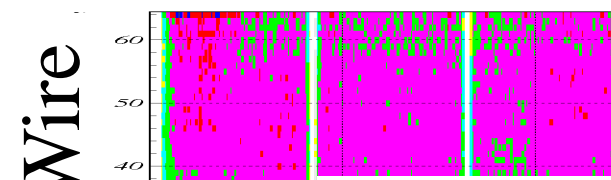

Example of uniform response

\#

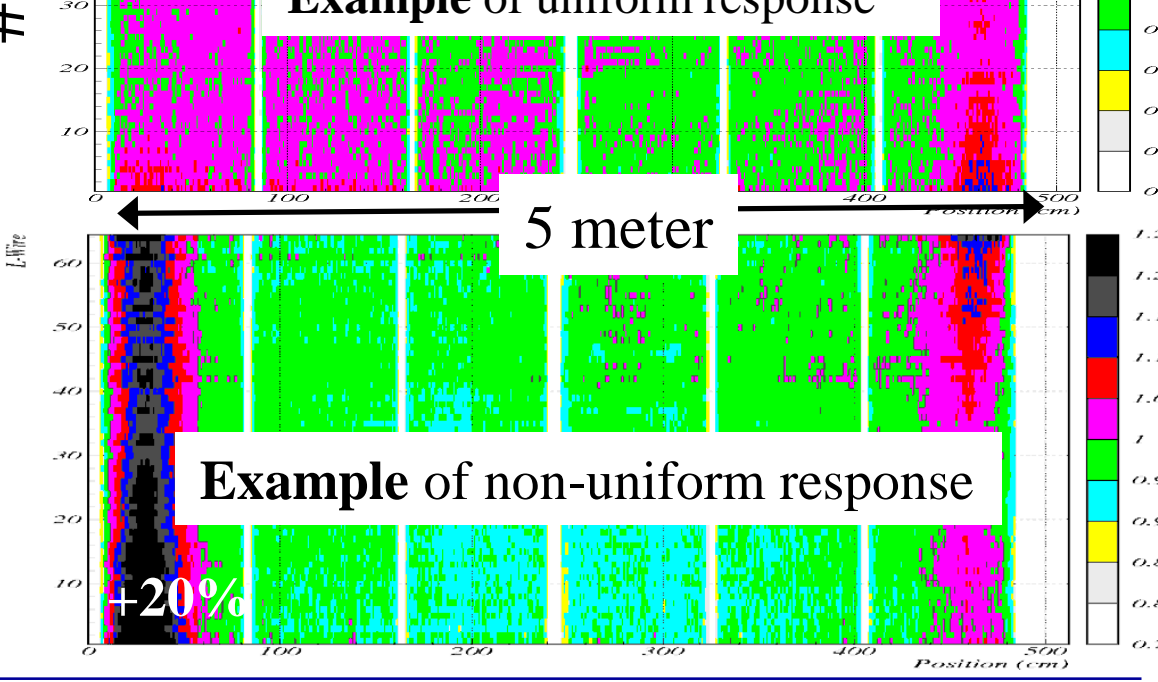

$11 / 18$ 


\section{Beam Test}
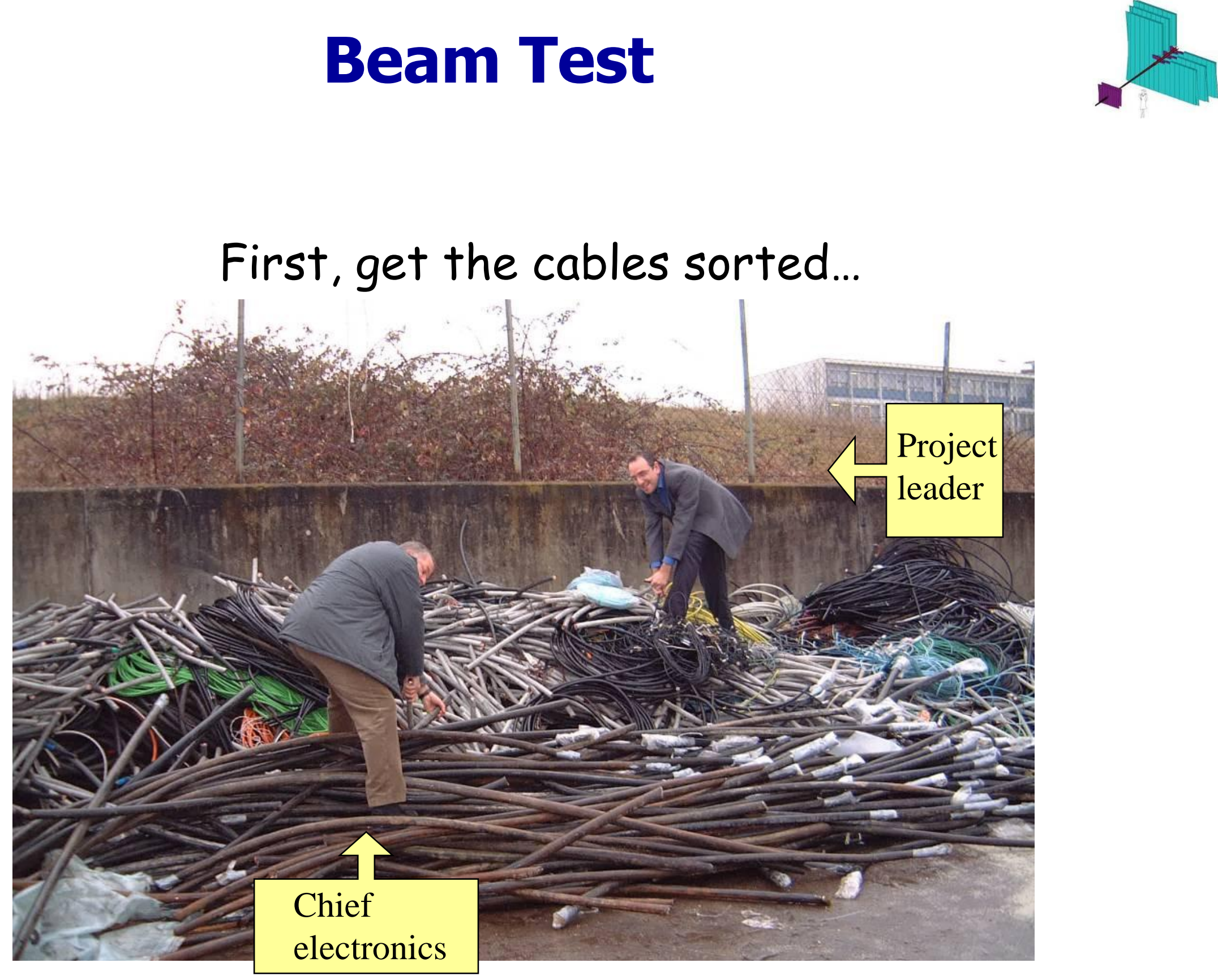


\section{Beam Test}

- Final detector modules

- Final prototype electronics

- $6 \mathrm{GeV}$ electrons at DESY

\section{Side View:}

\begin{tabular}{|l|l|l|}
\hline - & Efficiency \\
- & Resolution \\
- & Noise \\
- & Cross talk
\end{tabular} \mid $\begin{array}{ll}\text { vs } \\
\text { - Amplifier threshold } \\
\text { - Position along straw }\end{array}$

Outer Tracker Modules

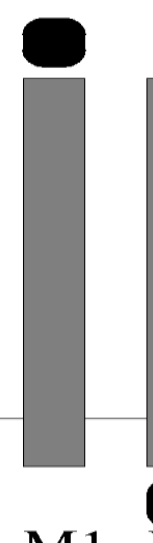

M1

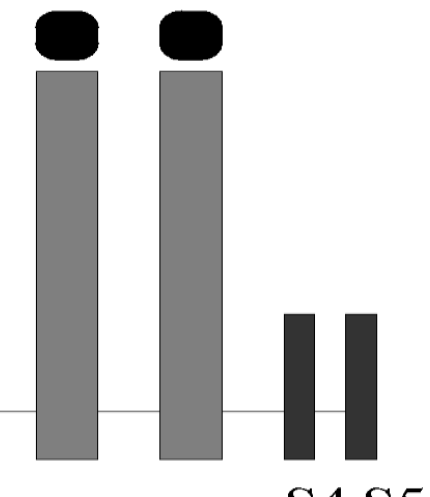
S1U009 siv002 S1U011 S1U005
$39 \mathrm{~cm}$

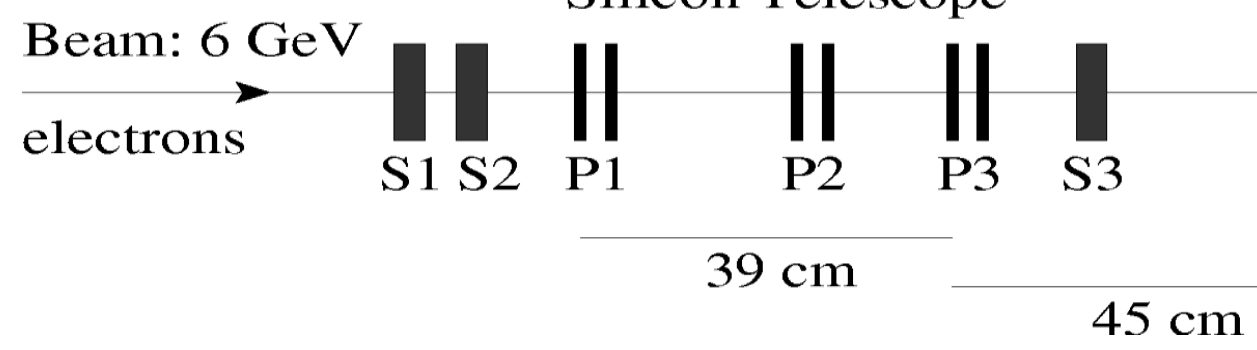

beam

\section{See: LHCb-2005-076}




\section{Beam Test}

rt-relation:

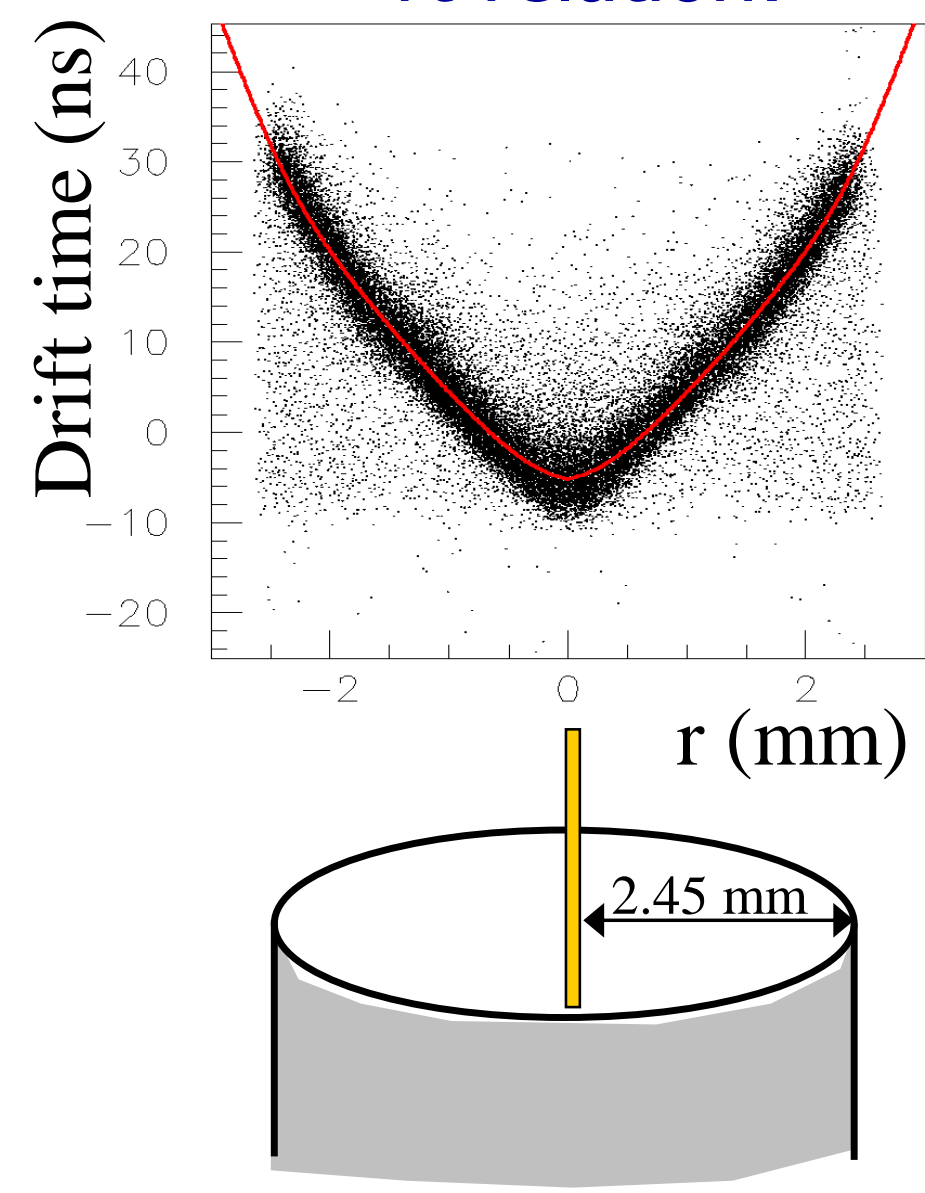

\section{efficiency profile:}
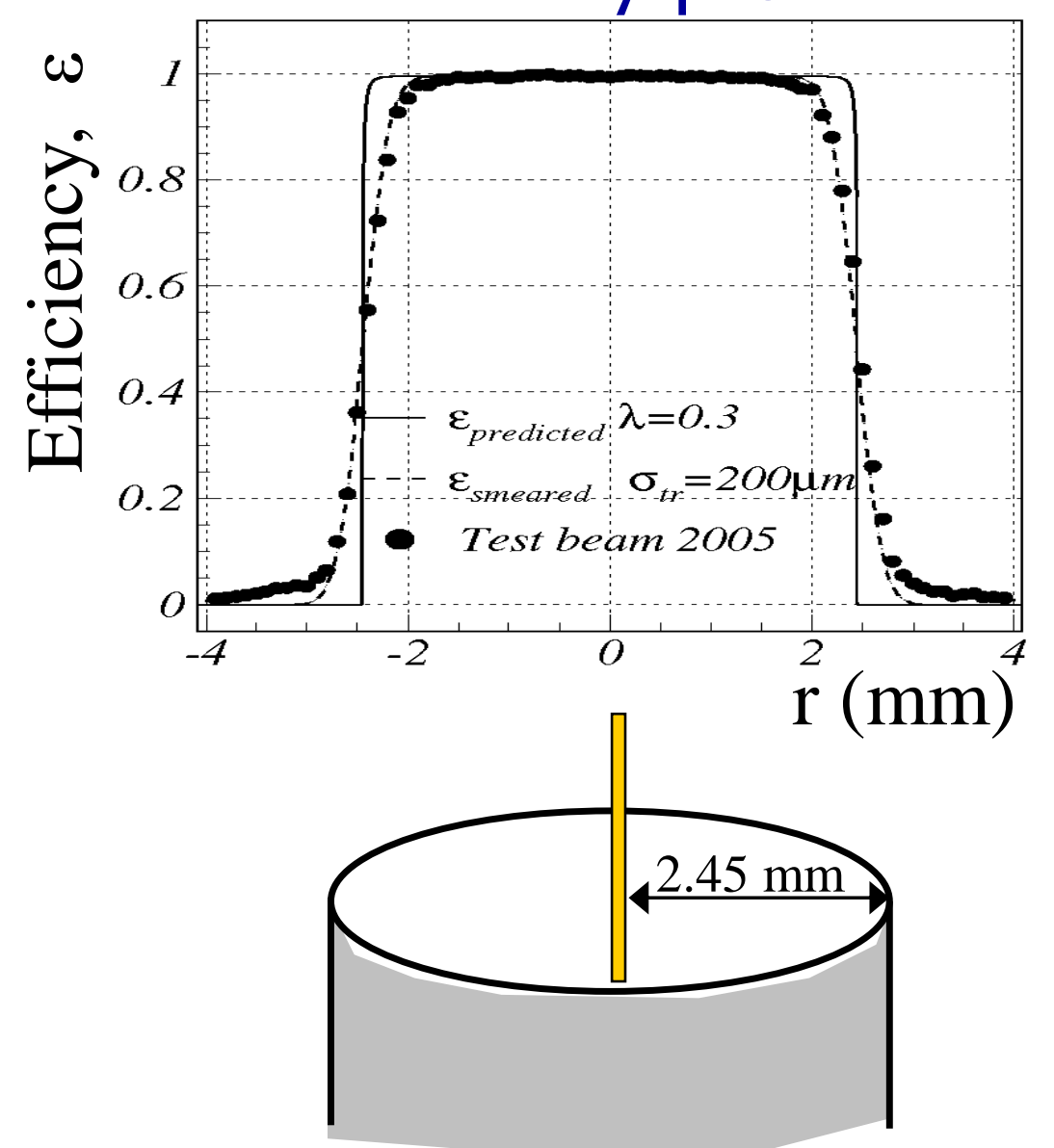

$\rightarrow$ Resolution and efficiency for different HV and amplifier threshold 


\section{Beam test: detector performance}

- Good efficiency and resolution for HV > $1520 \mathrm{~V}$

- $\varepsilon \approx 98 \%$

- $\sigma \approx 200 \mu \mathrm{m}$

- Corresponds to Gain>50,000
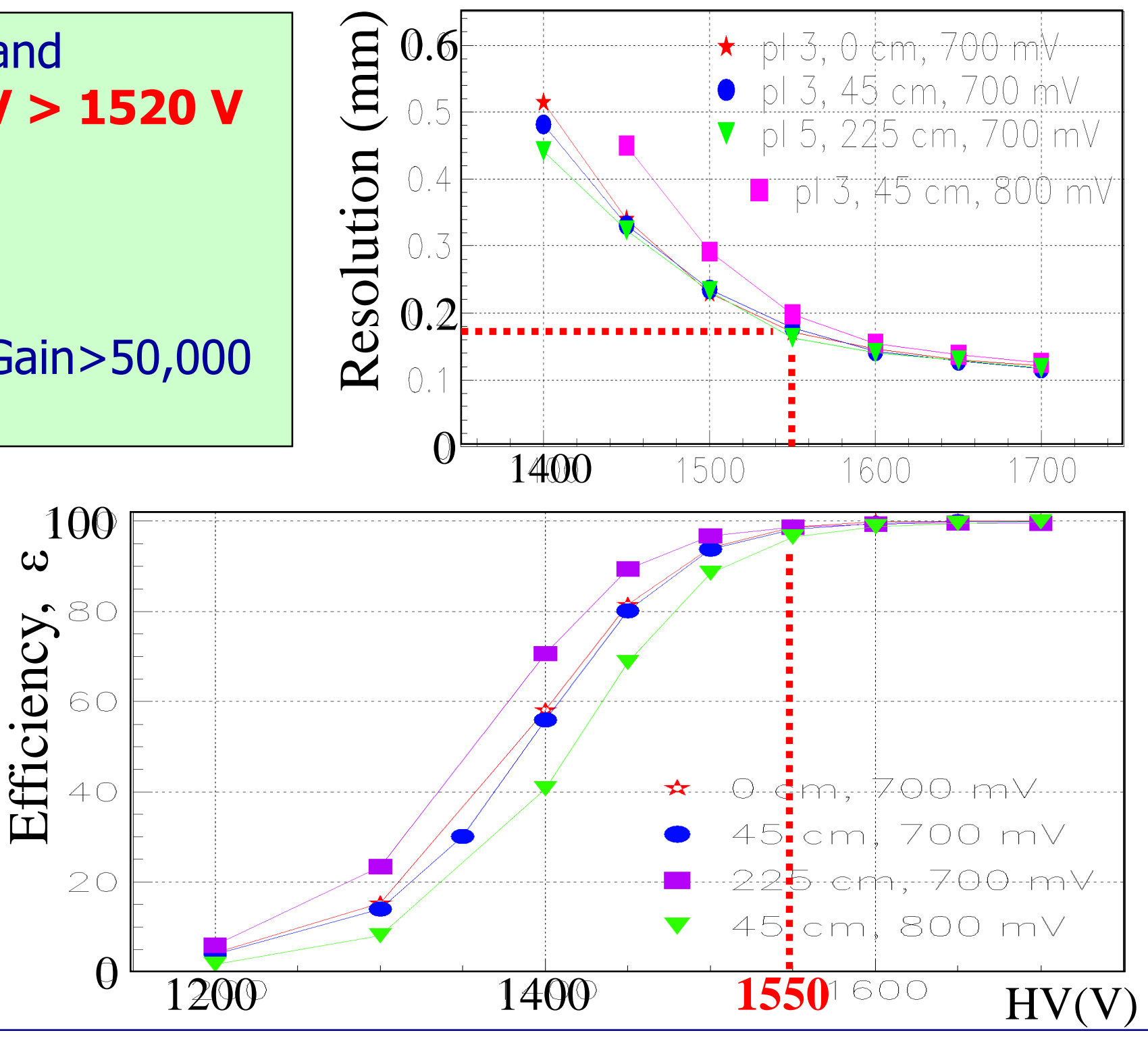


\section{Beam test: detector performance}

- Good efficiency and resolution for $700<$ thr $<900 \mathrm{mV}$

- $\varepsilon \approx 98 \%$

- $\sigma \approx 200 \mu \mathrm{m}$

- Corresponds to $3-5.5 \mathrm{fC}$

- Electronic noise at $750 \mathrm{mV}$ (= $3.5 \mathrm{fC})$ :

- $\approx 5 \mathrm{kHz} /$ straw

- $\approx 0.03 \%$ occupancy

- Acceptable
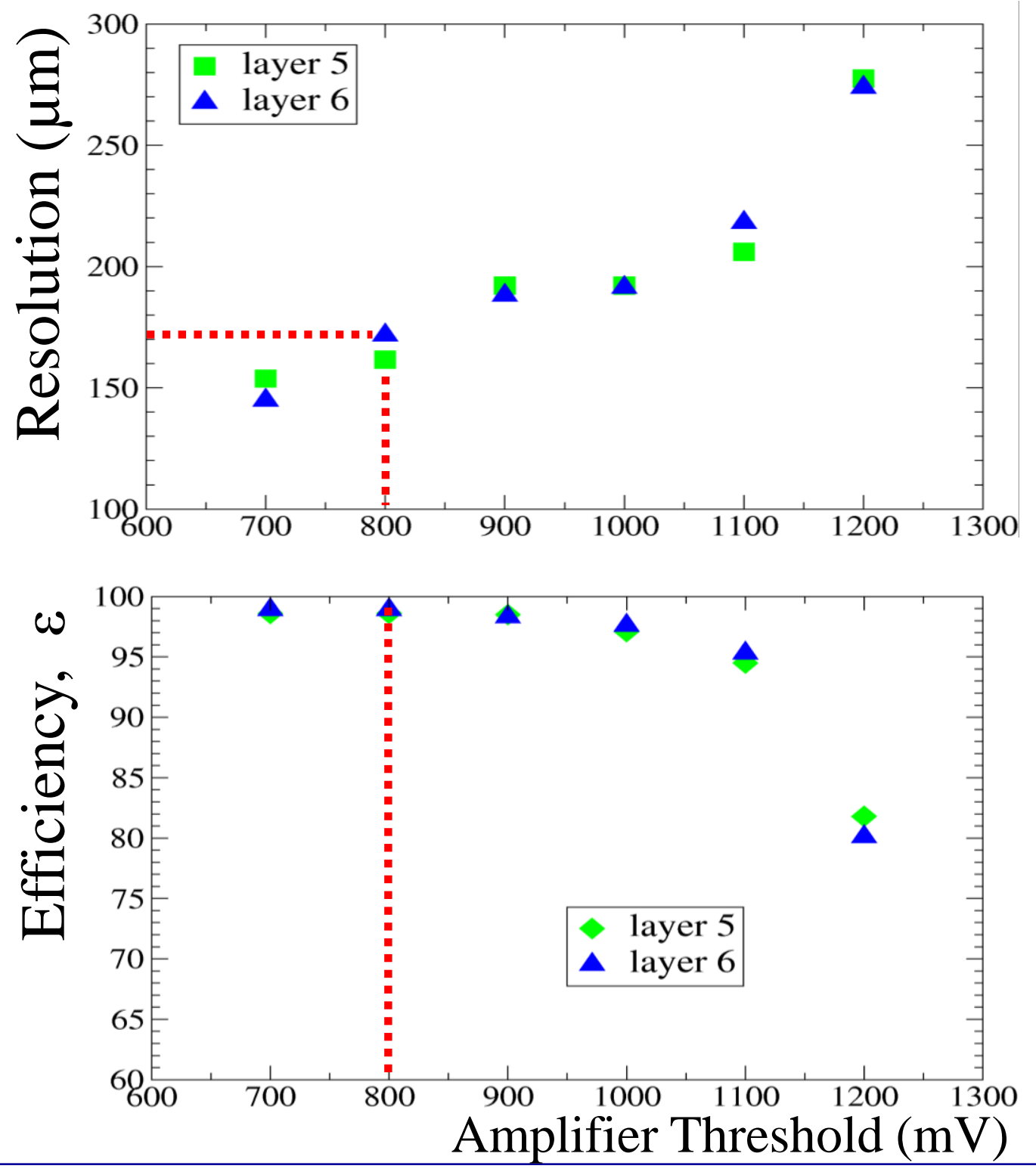


\section{Not everything is perfect...}

- Cross talk:

- Probability that neighbours fire

- $\approx 5 \%$ : acceptable

- Reflections:

- Straw not terminated

- New version of ASDBLR faster shaping

- $\rightarrow \approx 20 \%$ have additional after-pulse

- Effect much reduced depending on readout mode

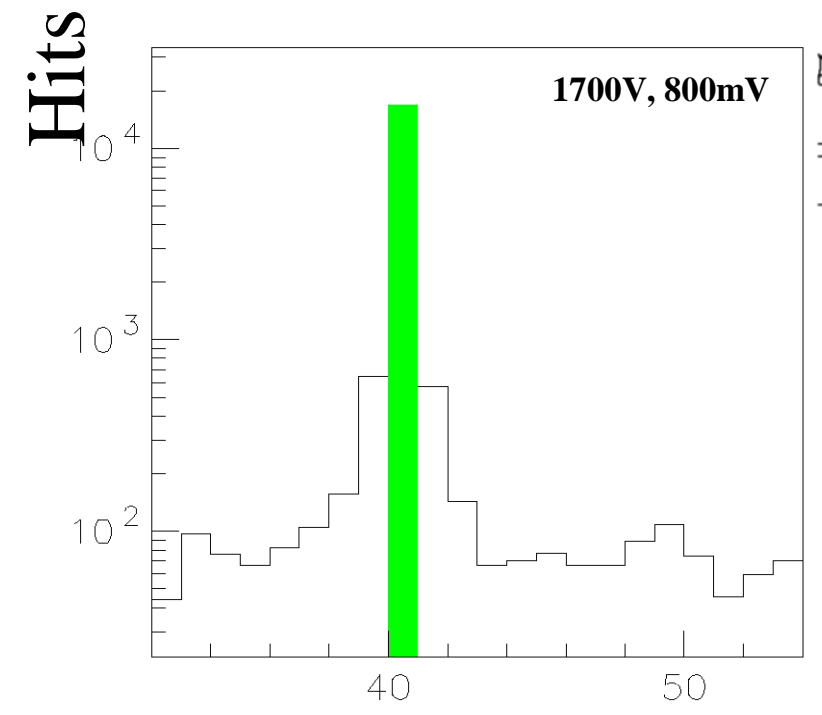

Channel

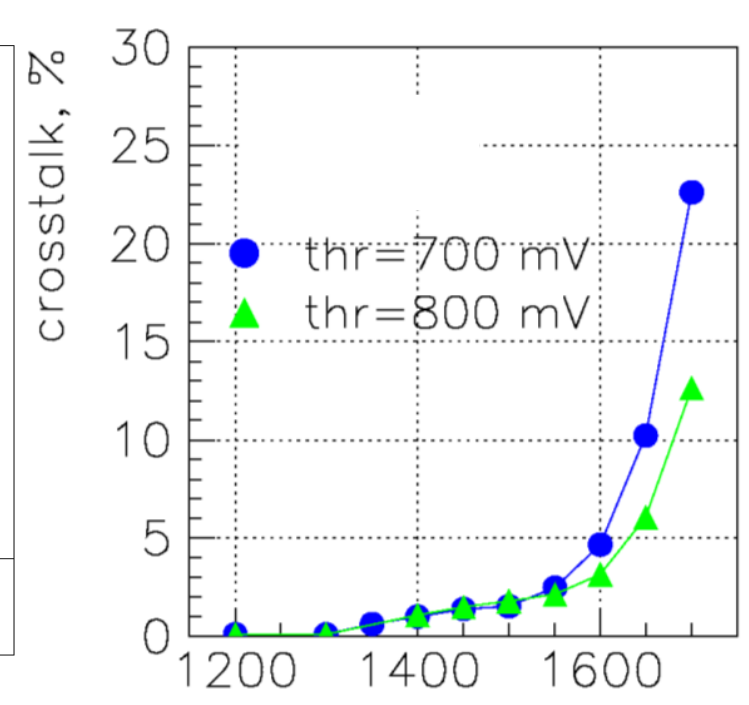

$\mathrm{HV}(\mathrm{V})$
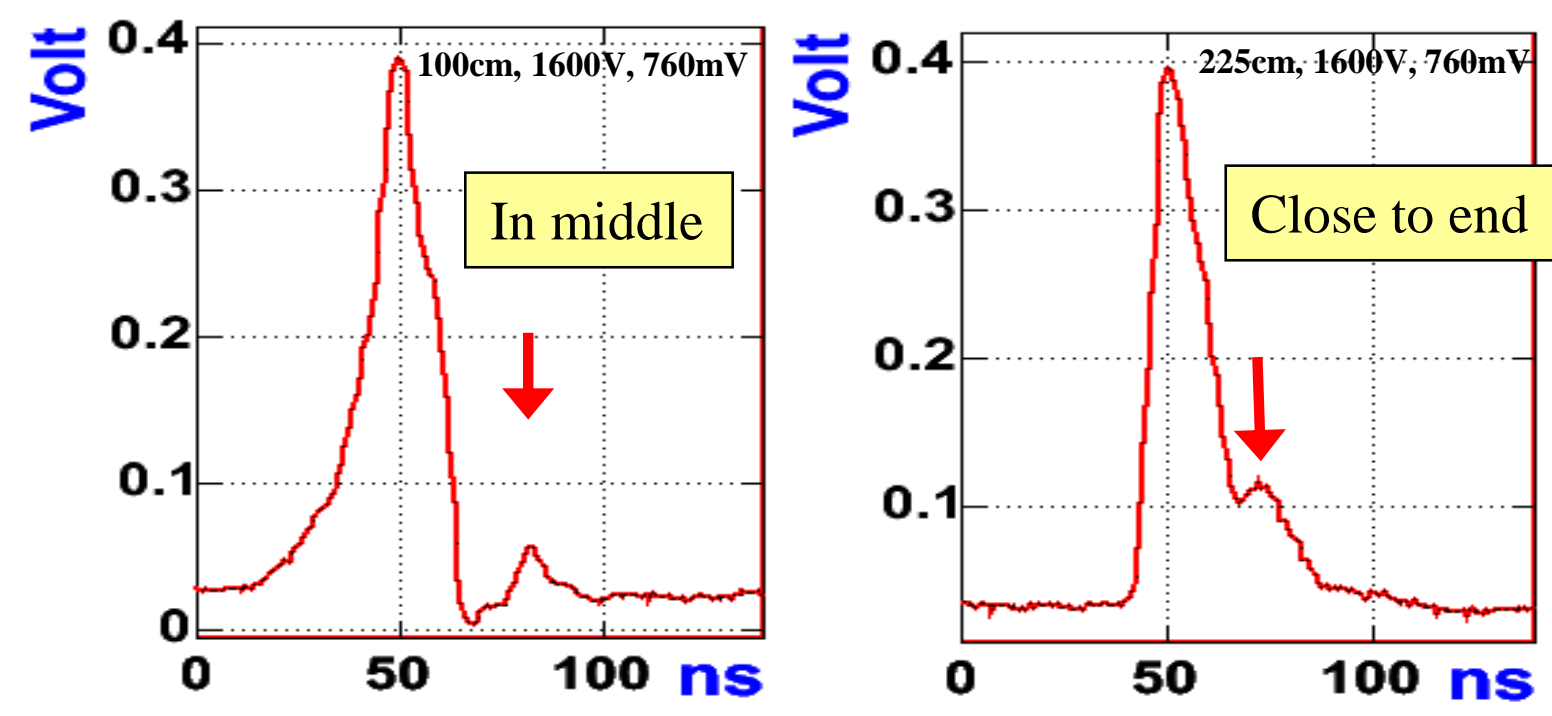

$17 / 18$ 


\section{Summary}

- Module production finished this week!

- Beam Test: detector characteristics determined:

- Good efficiency (>98\%)

- Good resolution $(<200 \mu \mathrm{m})$

- Noise level acceptable ( $5 \mathrm{kHz})$

- With some flexibility

- $1520-1600 \mathrm{~V}$

- 3-5 fC threshold

To do:

- Mechanical support

- Services

- HV, LV

- Gas

- Cooling

$\Rightarrow$ Install and commision in 2006 


\section{Backup: ageing studies}

- No news since report last year in the IEEE 2004 NSS-MIC conference (Oct 2004, Rome) by Sebastian Bachmann

- Session N39: Radiation Damage Effects II - Aging of Gaseous Detectors

- Title: Ageing Studies for the Straw Tube Detectors for the LHCb Outer Tracking System

\section{No ageing observed Studies continue}

